\title{
miR-142-3p targets AC9 to regulate sciatic nerve injury-induced neuropathic pain by regulating the cAMP/AMPK signalling pathway
}

\author{
XIAO LI $^{1}$, SHOUPENG WANG ${ }^{2}$, XIAOLI YANG ${ }^{3}$ and HONGJUN CHU ${ }^{2}$ \\ ${ }^{1}$ Department of Hand Surgery, Yantaishan Hospital, Yantai, Shandong 264000; ${ }^{2}$ Department of Orthopedics, \\ Zaozhuang Hospital of Zaozhuang Mining Group, Zaozhuang, Shandong 277100; ${ }^{3}$ Department of Pharmacy, \\ Yantai Affiliated Hospital of Binzhou Medical University, Yantai, Shandong 264000, P.R. China
}

Received July 13, 2020; Accepted November 11, 2020

DOI: $10.3892 /$ ijmm.2020.4824

\begin{abstract}
The aim of the present study was to investigate the effects of microRNA (miR)-142-3p on neuropathic pain caused by sciatic nerve injury in chronic compression injury (CCI) rats, and further investigate its mechanism. Rat experiments were divided into four parts in the study. In the first part, the rats were divided into the Sham and CCI groups. The expression of miR-142-3p, AC9 and cAMP were detected. In the second part, the rats were divided into the Sham, CCI, miR-142-3p mimic, mimic-negative control (NC), miR-142-3p small interfering RNA (siRNA) and siRNA-NC groups. The expression of cAMP and the levels of AMPK pathway-related proteins were detected. In the third part, the rats were randomly divided into Sham, CCI, AC9 mimic, mi-NC, AC9 siRNA and si-NC groups. Double luciferase reporter assay was used to analyse the targeting relationship between miR-142-3p and AC9. In the fourth part, the rats were divided into the Sham, CCI, miR-142-3p siRNA, AC9 mimic, miR-142-3p siRNA + AC9 siRNA, cAMP activator (Forskolin) and miR-142-3p siRNA + cAMP inhibitor groups. The expression of miR-142-3p was significantly increased while AC9 and cAMP expression significantly decreased in CCI rats. However, AC9 overexpression significantly increased the levels of cAMP protein. Luciferase reporter assay also proved that AC9 is the target gene of miR-142-3p. Moreover, miR-142-3p silencing was found to reduce neuropathic pain in CCI rats by upregulating the expression of AC9. It was also found that cAMP activation can relieve neuropathic pain and promote the expression of AMPK-related proteins in CCI rats. Silencing miR-142-3p can target AC9 to reduce the expression
\end{abstract}

Correspondence to: Dr Hongjun Chu, Department of Orthopedics, Zaozhuang Hospital of Zaozhuang Mining Group, 188 Shengli Road, Zaozhuang, Shandong 277100, P.R. China

E-mail: lixiaochuhongjun@sina.com

Key words: microRNA-142-3p, adenylate cyclase 9, sciatic nerve injury, cAMP/AMPK signalling pathway, TNF-a, IL-1 $\beta$ of inflammatory factors and neuropathic pain in CCI rats by increasing the expression of cAMP/AMPK pathway-related proteins.

\section{Introduction}

Neuropathic pain is a chronic type of pain caused by a primary lesion or dysfunction in both the central and peripheral nervous system (1). The causes of neuropathic pain are diverse, including alcoholism, amputation or spinal surgery (2). Nowadays, a variety of clinical treatment methods are tested, such as Aleve or Motrin, but serious side effects limit the scope and effect of treatment (3). Therefore, it is necessary to discover a novel and effective medical treatment for this condition.

MicroRNAs (miRs/miRNAs) are small non-coding RNAs that are 21-25 nucleotides in length (4). miRNAs play a post-transcriptional regulatory role by binding to the 3'-untranslated region (UTR) region or accidental 5'-UTR region of the target gene (5). Jiangpan et al demonstrated that the dysfunction of miRNAs is associated with various types of pathological conditions, including neuropathic pain (4). miR-142-3p is a highly conserved miRNA across vertebrates, which plays an essential role in the development and pathophysiology of the nervous system (5). Wang et al revealed that miR-142-3p expression was increased following sciatic nerve conditioning injury and is a target of adenylate cyclase 9 (AC9), and intracellular AC9 induced the upregulation of cAMP (6). However, Ouyang et al found that miR-142-3p was downregulated, instead of upregulated, in the spinal cord following chronic compression injury (CCI) (7). Therefore, the role of miR-142-3p in rats with sciatic nerve injury needs to be further clarified.

A previous study demonstrated that miR-142-3p is a novel regulator of inflammation that regulates proinflammatory mediators, including NF- $\kappa \mathrm{B}, \mathrm{TNF}-\mathrm{a}$ and IL-1 $\beta$ (8). Numerous studies have revealed that neuroinflammatory cytokines are involved in the maintenance and occurrence of neuropathic pain (9-11). Meanwhile, these inflammatory cytokines also accelerate the system of neuropathic pain, eventually leading to central and peripheral sensitization (10). The sciatic nerve tissue plays important roles in pain modulation and 
perception (11). Pain hypersensitivity in the sciatic nerve may result from neuronal sensitization and glial activation (11). It is therefore important to study the relationship between miR-142-3p and inflammatory cytokines in rats with sciatic nerve injury.

AMP-activated protein kinase (AMPK) is an essential sensor of cellular energy status and plays an important role in cellular energy balance (12). AMPK has been implicated in various diseases associated with energy metabolism, including neuropathic pain (13). In addition to the regulation of cellular energy metabolism, AMPK may mediate the pain response in preclinical pain models, and targeting AMPK is considered to be a novel strategy for the prevention and treatment of pain (14).

Based on previous studies, the present study hypothesized that miR-142-3p targets AC9 to regulate sciatic nerve injury-induced neuropathic pain via regulating the cAMP/AMPK signalling pathway.

\section{Materials and methods}

Animals. A total of 77 healthy male Sprague-Dawley rats, weighing 200-220 g [permission no. SCXK (Beijing) 20160006], aged 8 weeks, were purchased from Beijing Vital River Laboratory Animal Technology Co., Ltd. The rats were fed freely in a clean and quiet specific pathogen-free laboratory at $23 \pm 2^{\circ} \mathrm{C}, 55 \pm 5 \%$ humidity and a 12 -h light/dark cycle. The animal study protocol was in line with the National Institutes of Health (pub. no. 85-23, revised 1996). The study was approved by the Institutional Animal Care and Use Committee of Yantaishan Hospital (approval no. 20180705-0012).

Cell culture. 293T cells (cat. no. BNCC290119; BeNa Culture Collection) were cultured in DMEM (Gibco; Thermo Fisher Scientific, Inc.) supplemented with 10\% FBS (Sigma-Aldrich; Merck KGaA), $100 \mathrm{U} / \mathrm{ml}$ penicillin and $100 \mathrm{mg} / \mathrm{ml}$ streptomycin. All cells were incubated at $37^{\circ} \mathrm{C}$ with $5 \% \mathrm{CO}_{2}$. Cells in the logarithmic growth phase were collected and used for experiments.

Sciatic nerve injury model. A rat model was established using the CCI model method (15). Briefly, following the administration of $3 \%$ sodium pentobarbital $(50 \mathrm{mg} / \mathrm{kg})$ via intraperitoneal injection, the sciatic nerve of the right hind limb was exposed. Next, the sciatic nerve was ligated with a 4-0 catgut to tie four consecutive channels. The distance between each two channels was $1.0 \mathrm{~mm}$. The ligation intensity was appropriate when the nerve was slightly depressed, but the blood supply of the sciatic nerve was not blocked when observed under a microscope. Following surgery, the muscles and skin were sutured layer by layer and disinfected with iodophor, and the rats were fed in single cages.

Grouping and administration. In order to analyse the expression of miR-142-3p, AC9 and cAMP in the sciatic nerve of CCI rats, the rats were divided into two groups, with six rats per group: i) The sham group (Sham), in which the sciatic nerve of the right hind limb of the rats was exposed without ligation, the incisions were sutured layer by layer and ii) the CCI model group (CCI), in which the CCI model was established. In each group, three rats were sacrificed.
The intrathecal implantation was performed by inserting a PE-10 polyethylene catheter into the cisterna magna. For each intrathecal administration, miR-142-3p mimic (5'-UGUAGU GUUUCCUACUUUAUGGA-3'), mimic negative control (NC; 5'-CAGUACUUUUGUGUAGUACAA-3'), miR-142-3p small interfering RNA (siRNA; 5'-UCCAUAAAGUAGGAA ACACUACA-3'), siRNA control (5'-CAGUACUUUUGU GUAGUACAA-3'), expression plasmid AC9 (5'-UUCUUU UCUUAAAAAUGUGAUGG-3'), plasmid control (5'-CAG UACUUUUGUGUAGUACAA-3'), AC9 siRNA (5'-AAGGAG ATGGTGAACATGAGA-3') and AC9 siRNA control (5'-CAG UACUUUUGUG UAGUACAA-3') (all from Shanghai GenePharma Co., Ltd.) was injected into the intrathecal catheter using a microinjection syringe.

To determine the role of miR-142-3p in sciatic nerve injury of CCI rats, the rats were divided into six groups, with five rats per group; i) The sham group (Sham), in which an intrathecal injection of $10 \mu \mathrm{l}$ normal saline was administered daily for 3 consecutive days; ii) the CCI model group (CCI), in which an intrathecal injection of $10 \mu \mathrm{l}$ normal saline was administered daily for 3 consecutive days; iii) the miR-142-3p mimic treatment group (miR-142-3p mimic), in which the CCI model was established and then the rats were intrathecally injected with $10 \mu \mathrm{l}$ $10 \mu \mathrm{M}$ miR-142-3p mimic (16) every day for 3 consecutive days; iv) the miR-142-3p mimic NC group (mimic-NC), in which the $\mathrm{CCI}$ model was established and then the rats were intrathecally injected with $10 \mu 110 \mu \mathrm{M}$ mimic NC daily for 3 consecutive days; v) the miR-142-3p siRNA treatment group (miR-142-3p siRNA), in which the CCI model was established and then the rats were intrathecally injected with $10 \mu 110 \mu \mathrm{M}$ miR-142-3p siRNA every day for 3 consecutive days and vi) the miR-142-3p siRNA NC group (siRNA-NC), in which the CCI model was established and then the rats were intrathecally injected with $10 \mu 110 \mu \mathrm{M}$ siRNA NC daily for 3 consecutive days.

To investigate the role of AC9 in sciatic nerve injury in CCI rats, the rats were divided into four groups, with five rats per group: i) The expression plasmid AC9 treatment group (pcDNA-AC9), in which the CCI model was established and then the rats were intrathecally injected with $3 \mu 10.005 \mathrm{mg} / \mu \mathrm{l}$ pcDNA-AC9 (16) every day for 3 consecutive days; ii) the plasmid control group (pc-NC), in which the CCI model was established and then the rats were intrathecally injected with $3 \mu 10.005 \mathrm{mg} / \mu \mathrm{l}$ plasmid control vector daily for 3 consecutive days; iii) the AC9 siRNA treatment group (AC9 siRNA), in which the CCI model was established and then the rats were intrathecally injected with $3 \mu 10.005 \mathrm{mg} / \mu \mathrm{l}$ AC9 siRNA every day for 3 consecutive days and iv) the AC9 siRNA control group (si-NC), in which the CCI model was established and then the rats were intrathecally injected with $3 \mu 10.005 \mathrm{mg} / \mu \mathrm{l}$ of siRNA NC daily, for 3 consecutive days.

To reveal the effects of the interaction between AC9 and miR-142-3p on sciatic nerve injury-induced neuropathic pain, the rats were further divided into three groups, with five rats per group: i) The AC9 siRNA and miR-142-3p siRNA treatment group (miR-142-3p siRNA + AC9 siRNA), in which the CCI model was established and then the rats were intrathecally injected with $3 \mu \mathrm{l} 0.005 \mathrm{mg} / \mu \mathrm{l}$ AC9 siRNA and $10 \mu \mathrm{l} 10 \mu \mathrm{M}$ miR-142-3p siRNA every day for 3 consecutive days; ii) the cAMP activator group (Forskolin), in which the CCI model was established and then the rats were intrathecally injected 
with $3 \mu \mathrm{M}$ forskolin (17) (cat. no. S1612-5 mg; Beyotime Institute of Biotechnology) daily for 3 consecutive days; iii) the miR-142-3p siRNA and cAMP inhibitor treatment group (miR-142-3p siRNA + SQ22536), in which the CCI model was established and then the rats were intrathecally injected with $10 \mu 110 \mu \mathrm{M}$ miR-142-3p siRNA and $500 \mu \mathrm{M}$ SQ22536 (17) (MB3735; Dalian Meilun Biology Technology Co., Ltd.) every day for 3 consecutive days.

Measurement of paw withdrawal mechanical threshold(PWMT) and paw withdrawal thermal latency (PWTL) in rats. PWMT and PWTL values were measured once a day before surgery, which constituted the preoperative values. The two indicators were tested again on days 4, 7, 10, 14 and 21 following surgery, according to Hargreaves et al (18) and Shao et al (19). Briefly, rats were individually placed in a $1-\mathrm{mm}$ thick plastic chamber $\left(7 \times 9 \times 11 \mathrm{~cm}^{3}\right)$ with a smooth glass surface on the base for PWTL measurement. The rats underwent a familiarization period $45 \mathrm{~min}$ before the behavioural tests. To avoid tissue damage, an automatic 20 sec cut-off value was set. The hind paw of each right posterior limb was repeated three times with a 5-min interval. Mechanical allodynia was determined by foot withdrawal with Von Frey filaments (North Coast Medical Inc.). Briefly, rats were laid individually on a wire mesh floor in a $20 \times 25 \times 15-\mathrm{cm}^{3}$ plastic box with a 45-min adoption before the text. The filaments were perpendicularly placed to the plantar surface of the hind paws with increasing forces until the maximum stimulus $(4.0 \mathrm{~g})$ or 10 positive responses. The mechanical allodynia was determined as the force of a minimum detectable withdrawal on $50 \%$ of the tests at the same force level.

Sample collection. On the 10th day following surgery, the rats were injected intraperitoneally with $3 \%$ pentobarbital sodium $(50 \mathrm{mg} / \mathrm{kg}$ ) and then perfused with $200 \mathrm{ml}$ old saline. The L4-6 sciatic nerve on the right side of the rats was removed. Some parts were fixed with $4 \%$ paraformaldehyde at room temperature for $24 \mathrm{~h}$ for immunohistochemistry, while others were stored in liquid nitrogen for western blotting and reverse transcription-quantitative PCR (RT-qPCR) analysis.

$R T$ - $q P C R$. Total RNA was extracted using TRIzol ${ }^{\circledR}$ reagent (cat. no. 15596018; Invitrogen; Thermo Fisher Scientific, Inc.). cDNA was then synthesized using TaqMan reverse transcription kits (Thermo Fisher Scientific, Inc.). RT-PCR was performed using Mastercycler ${ }^{\circledR}$ Nexus X2 (Eppendorf) with the SYBR Premix Ex Taq II kit (cat. no. RR820A; Takara Bio, Inc.). The conditions were $95^{\circ} \mathrm{C}$ for $15 \mathrm{sec}, 60^{\circ} \mathrm{C}$ for $60 \mathrm{sec}$, and $72^{\circ} \mathrm{C}$ for $40 \mathrm{sec}$ ( 35 cycles). Data were processed by the $2^{-\Delta \Delta \mathrm{Cq}}$ method (20).

The primer sequences were designed as follows: miR-142-3p forward, 5'-CTCCTGTAGTGTTTCCTAC-3' and reverse, 5'-GACTGTTCCTCTCTTCCTC-3'; U6 forward, 5'-TCGCTTCGGCAGCACATA-3' and reverse, 5'-TTTGCG TGTCATCCTTGC-3'; AC9 forward, 5'-AACAGCACCAAG GCTTCTGGAGGAC-3' and reverse, 5'-TCTTGAACCTCA GCGGAAGGAGAGC-3' and GAPDH forward, 5'-CATCAC TGCCACCCAGAAGACTG-3' and reverse, 5'-ATGCCAGTG AGCTTCCCGTTCAG-3'.

ELISA. Sciatic nerve samples were prepared using $0.1 \mathrm{~N} \mathrm{HCl}$ and centrifuged at $10,000 \mathrm{x} \mathrm{g}$ at $4^{\circ} \mathrm{C}$ for $20 \mathrm{~min}$ to eliminate impurities. The acquired supernatant was neutralized with $1 \mathrm{~N}$ NaOH.cAMP ELISA kit (cat. no. BP-E30574-2; R\&D Systems, Inc.) was used to detect the levels of cAMP in the supernatant according to the manufacturer's instructions.

The sciatic nerve samples were treated with lysis buffer (cat. no. R0010; Beijing Solarbio Science \& Technology Co., Ltd.) and benzylsulfonyl fluoride, incubated at $4^{\circ} \mathrm{C}$ for $10 \mathrm{~min}$, then centrifuged at $10,000 \mathrm{x} \mathrm{g}$ at $4^{\circ} \mathrm{C}$ for $15 \mathrm{~min}$ to collect the supernatant. The levels of pro-inflammatory cytokines TNF- $\alpha$ (cat. no. RAT00) and IL-1 $\beta$ (cat. no. RLB00) were measured by ELISA kits (R\&D Systems, Inc.) according to the manufacturer's instructions.

Immunohistochemistry. Following routine sectioning, specimens embedded in paraffin were baked at $65^{\circ} \mathrm{C}$ for $100 \mathrm{~min}$, dewaxed with xylene and hydrated with a serial ethanol solution. The solution was inactivated by adding $3 \% \mathrm{H}_{2} \mathrm{O}_{2}$ methanol solution for $20 \mathrm{~min}$ at room temperature, heat-fixed with high temperature antigen in citrate buffer $(\mathrm{pH} \mathrm{6.0)}$ for $10 \mathrm{~min}$ and blocked with 5\% BSA (cat. no. SW3015; Beijing Solarbio Science \& Technology Co., Ltd.) for $20 \mathrm{~min}$ at room temperature. Rabbit anti-rat IL-1 $\beta$ (1:1,000; cat. no. ab9722; Abcam) polyclonal antibody and TNF-a (1:1,000; cat. no. ab66579; Abcam) polyclonal antibody were added and incubated overnight at $4^{\circ} \mathrm{C}$. Washing with phosphate buffer saline (PBS) at room temperature, $5 \mathrm{~min}, 3$ times, the samples were incubated with goat anti-rabbit HRP-conjugated secondary antibody (1:1,000; cat. no. ABIN101988; antibodies-online GmbH). Slides were stained with diaminobenzidine (Beyotime Institute of Biotechnology) and re-dyed with haematoxylin. After dehydrating with gradient ethanol solution, the slices were sealed with neutral gum. Results were observed at x400 magnification under an upright microscope (CX43; Olympus Corporation) and analysed by ImageJ 5.0 software (National Institutes of Health). Cells positive for IL-1 $\beta$ and TNF-a expression were stained brown. Positive cells $(\%)=$ numbers of positive cells/numbers of total cells $\times 100 \%$.

Western blotting. Total protein from sciatic nerve tissues were extracted using RIPA buffer (Beyotime Institute of Biotechnology) and then quantified using a Pierce ${ }^{\mathrm{TM}}$ BCA Protein Assay kit (Thermo Fisher Scientific, Inc.). A total of $40 \mu \mathrm{g}$ protein/lane was separated via $10 \%$ SDS-PAGE (Mini-Protean-3; Bio-Rad Laboratories, Inc.) and transferred to a polyvinylidene difluoride membrane (EMD Millipore). The membrane was blocked with $5 \%$ skimmed milk powder solution for $1 \mathrm{~h}$ at room temperature and then incubated with the following primary antibodies at $4^{\circ} \mathrm{C}$ overnight: Anti-rabbit phosphorylated (p)-AMPKa (1:1,000; cat. no. 4184; Cell Signaling Technology, Inc.), AMPKa (1:1,000; cat. no. 2532; Cell Signaling Technology, Inc.), p-NF-кB p65 (1:1,000; cat. no. 3033, Cell Signaling Technology, Inc.), NF-кB p65 (1:1,000, cat. no. 4746; Cell Signaling Technology, Inc.), p-IкBa (1:1,000; cat. no. vk10582; Guangzhou Weiboxin Biotechnology Co., Ltd.), IкBa (1:1,000; cat. no. A2457; ABclonal Biotech Co., Ltd.), AC9 (1:1,000; cat. no. ab191423; Abcam) and GAPDH (1:1,000; cat. no. AC033; ABclonal Biotech Co., Ltd.). The membranes were then incubated with goat anti-rabbit immunoglobulin $\mathrm{G}$ secondary antibody (1:1,000; cat. no. ABIN101988; antibodies-online $\mathrm{GmbH})$ at 

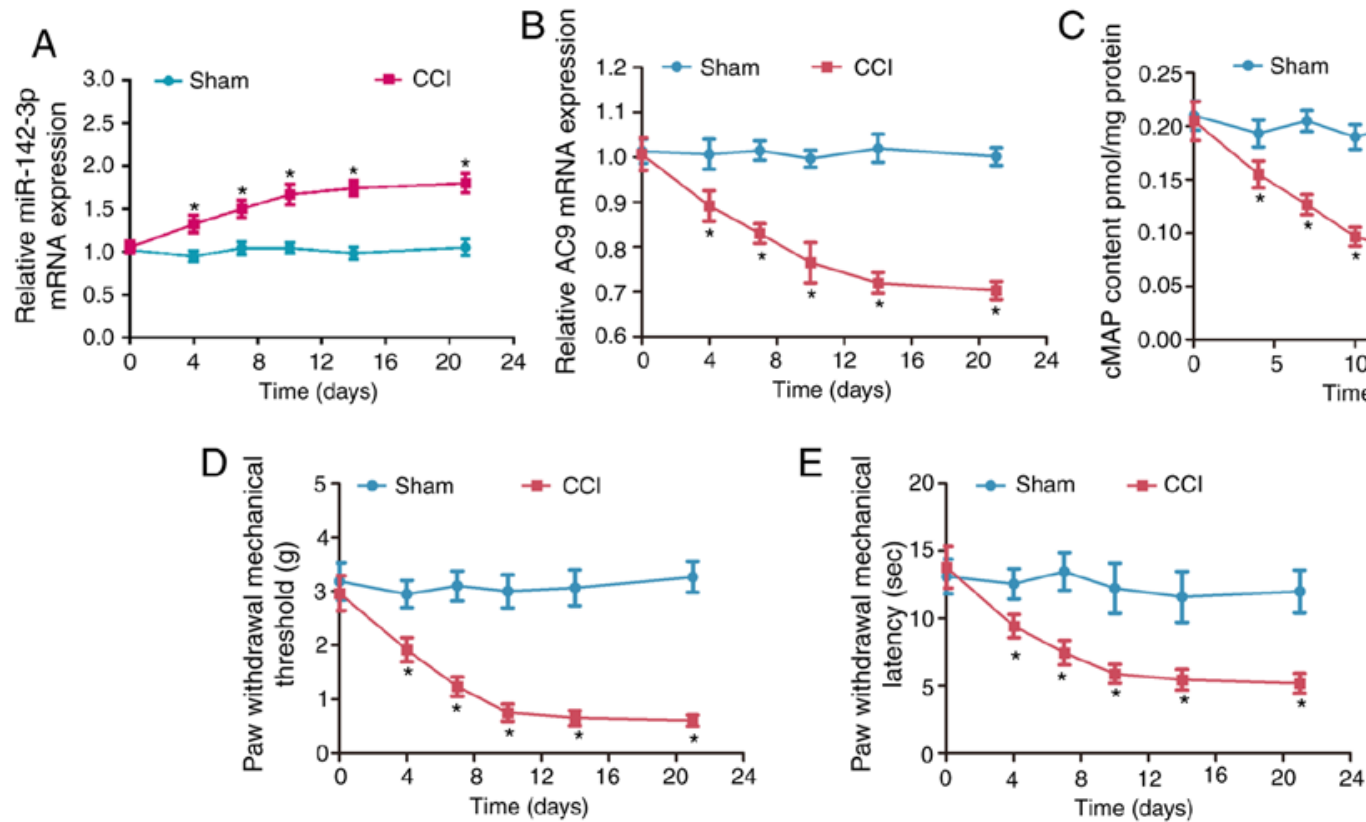

Figure 1. Expression of miR-142-3p, AC9 and cAMP and changes in PWMT and PWTL in sciatic nerve injury rats. The expression of (A) miR-142-3p and (B) AC9 mRNA was analysed by reverse transcription-quantitative PCR. (C) cAMP levels were analysed by ELISA. The changes in (D) PWMT and (E) PWTL were measured in rats. * $\mathrm{P}<0.05$ vs. sham group. Each experiment was repeated six times. miR, microRNA; PWMT, paw withdrawal mechanical threshold; PWTL, paw withdrawal thermal latency; CCI, chronic compression injury; AC9, adenylate cyclase 9.

room temperature for $2 \mathrm{~h}$ and treated with enhanced chemiluminescence solution (Thermo Fisher Scientific, Inc.). GAPDH was used as the internal reference and the integrated density values of protein bands were quantitatively analysed by ImageJ 5.0 software (National Institutes of Health).

Target prediction for miR-142-3p. Potential target genes of miR-142-3p were predicted by TargetScan (targetscan.org/), which is a web-based resource used for the prediction of biological targets of miRNAs by searching for the presence of 8-, 7- and 6-mer sites that match the seed region of each miRNA.

Dual-luciferase reporter assay. The 3'-UTR regions of AC9 containing the predicted miR-142-3p specific binding sites were amplified by RT-PCR and cloned into the pmirGLO firefly luciferase reporter vector (Promega Corporation) to obtain the wild-type luciferase reporter plasmids (wt-AC9). In order to generate mutant reporter plasmids (mut-AC9), certain nucleotides in AC9 3'-UTR lacking miR-142-3p binding sites were mutated using RT-PCR. The constructed luciferase reporter plasmids were separately co-transfected with $50 \mathrm{nM}$ miR-142-3p mimic or mimic NC into 293T cells using Lipofectamine ${ }^{\circledR} 2000$ (Invitrogen; Thermo Fisher Scientific, Inc.). Cells were lysed using lysis buffer (cat. no. R0010; Beijing Solarbio Science \& Technology Co., Ltd.) for $15 \mathrm{~min}$ at room temperature and luciferase activities of gene plasmid and phRL-TK were assayed at $48 \mathrm{~h}$ post-transfection using a Dual-Luciferase Reporter Assay system (Promega Corporation) according to the manufacturer's instructions.

Statistical analysis. All data were analysed by SPSS 19.0 software (IBM Corp.) and presented as the mean \pm SD. Comparisons between groups were conducted by t-test. In addition, multiple comparisons were performed using one-way ANOVA and Tukey's post hoc test. $\mathrm{P}<0.05$ was considered to indicate a statistically significant difference.

\section{Results}

Expression of miR-142-3p, AC9 and cAMP following sciatic nerve injury. To characterize the potential role of mir-142-3p in neuropathic pain, the expression of miR-142-3p (Fig. 1A) and AC9 (Fig. 1B) were detected by RT-qPCR. Compared with the sham group, miR-142-3p was significantly upregulated and AC9 mRNA was significantly downregulated in the CCI group $(\mathrm{P}<0.05)$. cAMP levels significantly decreased in the CCI group compared with the sham group $(\mathrm{P}<0.05$; Fig. 1C). PWMT and PWTL values were significantly decreased in the CCI group compared with the sham group $(\mathrm{P}<0.05$; Fig. $1 \mathrm{D}$ and $\mathrm{E})$. These data showed that the miR-142-3p expression was significantly increased, but the AC9 and cAMP expression was clearly decreased in the sciatic nerve of CCI rats.

Effects of miR-142-3p on sciatic pain and the AMPK pathway. To investigate the association between miR-142-3p and neuropathic pain, miR-142-3p mimic and siRNA were injected intrathecally, and the expression of miR-142-3p in each group is shown in Fig. 2A. miR-142-3p expression was significantly increased in the miR-142-3p mimic group and significantly decreased in the miR-142-3p siRNA group when compared with their respective $\mathrm{NC}$ groups $(\mathrm{P}<0.05)$. In addition, the cAMP content was significantly reduced in the miR-142-3p mimic group and increased in the miR-142-3p siRNA group compared with their respective $\mathrm{NC}$ groups $(\mathrm{P}<0.05$; Fig. $2 \mathrm{~B})$.

Meanwhile, the PWMT and PWTL values were used to study the role of the miR-142-3p expression in sciatica pain. The results indicated that miR-142-3p inhibition effectively increased 
A
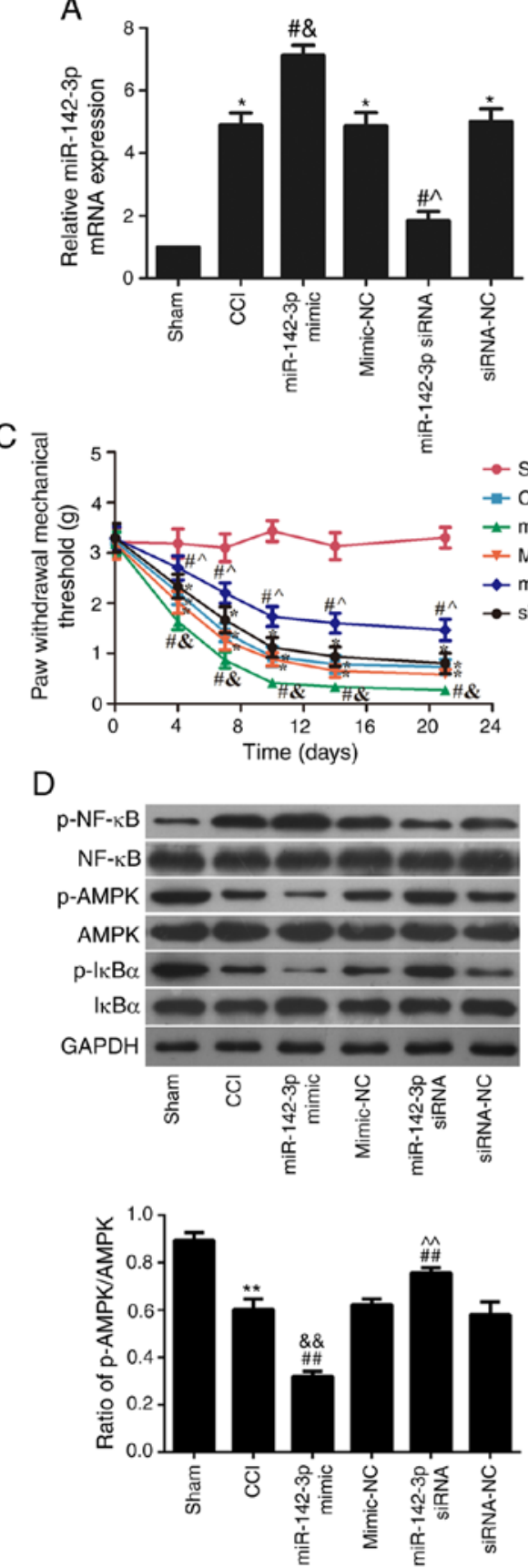

B
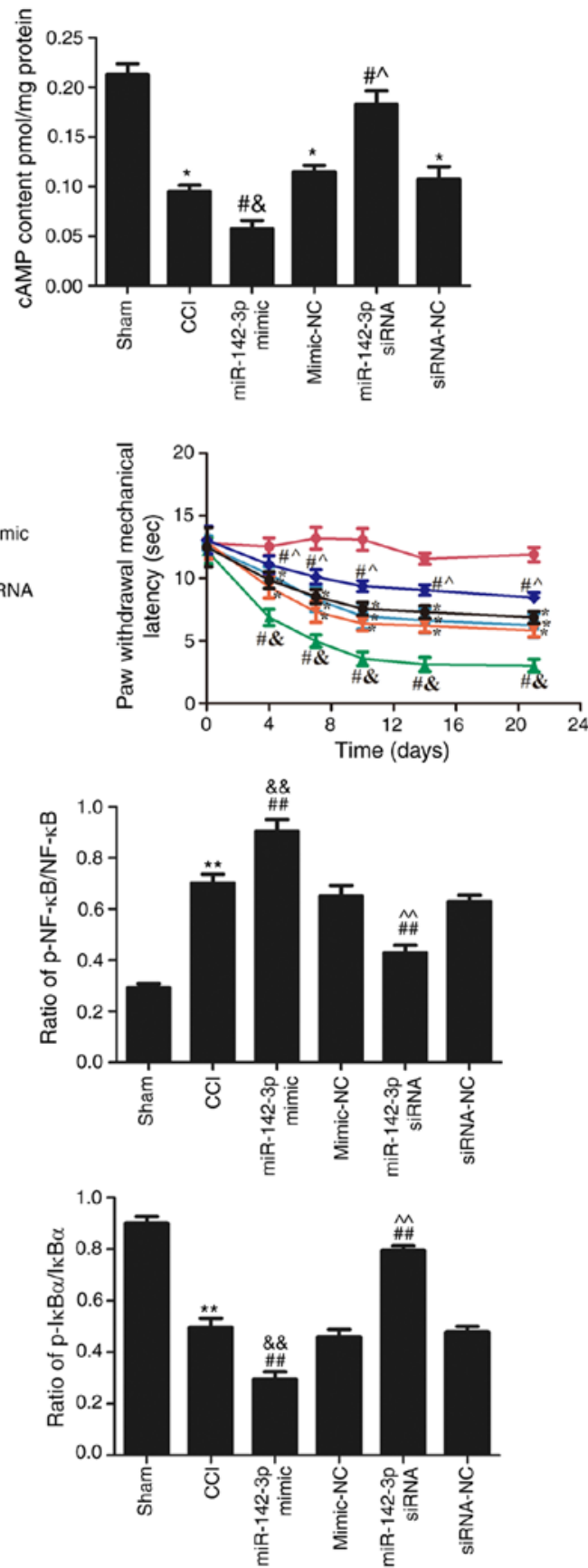

Figure 2. Effects of miR-142-3p on sciatic pain and the AMPK pathway. (A) miR-142-3p expression was analysed by reverse transcription-quantitative PCR (B) cAMP levels were analysed by ELISA. (C) The changes in paw withdrawal mechanical threshold and paw withdrawal thermal latency were measured in rats. (D) The levels of p-AMPK/AMPK, p-NF- $\kappa \mathrm{B} / \mathrm{NF}-\kappa \mathrm{B}$ and $\mathrm{p}-\mathrm{I} \kappa \mathrm{Ba} / \mathrm{I} \kappa \mathrm{Ba}$ were analysed by western blotting. ${ }^{*} \mathrm{P}<0.05$ and ${ }^{* *} \mathrm{P}<0.01$ vs. sham group. ${ }^{\#} \mathrm{P}<0.05$ and ${ }^{\# \#} \mathrm{P}<0.05$ vs. CCI group. ${ }^{\&} \mathrm{P}<0.05$ and ${ }^{\& \&} \mathrm{P}<0.05$ vs. mimic-NC group. ${ }^{\wedge} \mathrm{P}<0.05$ and ${ }^{\wedge \wedge} \mathrm{P}<0.05$ vs. siRNA-NC group. Each experiment was repeated five times. miR, microRNA; AMPK, AMP-activated protein kinase; siRNA, small interfering RNA; NC, negative control; CCI, chronic compression injury; p, phosphorylated.

the PWMT and PWTL values following surgery compared with the siRNA-NC group ( $\mathrm{P}<0.05$; Fig. 2C). However, miR-142-3p overexpression reduced the PWMT and PWTL values following surgery compared with the mimic-NC group $(\mathrm{P}<0.05$; Fig. $2 \mathrm{C})$. These results suggested that miR-142-3p downregulation suppressed sciatic pain in CCI rats.

The levels of $\mathrm{p}-\mathrm{AMPK} / \mathrm{AMPK}, \mathrm{p}-\mathrm{NF}-\kappa \mathrm{B} / \mathrm{NF}-\kappa \mathrm{B}$ and $\mathrm{p}-\mathrm{I} \kappa \mathrm{Ba} / \mathrm{I} \kappa \mathrm{Ba}$ in the sciatic nerve were detected using western blotting (Fig. 2D). Compared with the sham group, the phosphorylation levels of AMPK and $\mathrm{I} \kappa \mathrm{Ba}$ were significantly reduced $(\mathrm{P}<0.05)$, while the phosphorylation levels of $\mathrm{NF}-\kappa \mathrm{B}$ was significantly increased $(\mathrm{P}<0.05)$ in the CCI group. Following miR-142-3p mimic transfection, compared with the CCI group, the phosphorylation levels of AMPK and I $\mathrm{Ba}$ were significantly reduced $(\mathrm{P}<0.05)$, while the phosphorylation levels of $N F-\kappa B$ was further increased $(\mathrm{P}<0.05)$. However, transfection of miR-142-3p siRNA significantly elevated the phosphorylation levels of AMPK and I $\mathrm{Ba}$ and inhibited the phosphorylation levels of NF- $\mathrm{B}$ compared with the $\mathrm{CCI}$ group $(\mathrm{P}<0.05)$.

Effects of miR-142-3p on inflammatory cytokine content following sciatic nerve injury. The effects of miR-142-3p on 


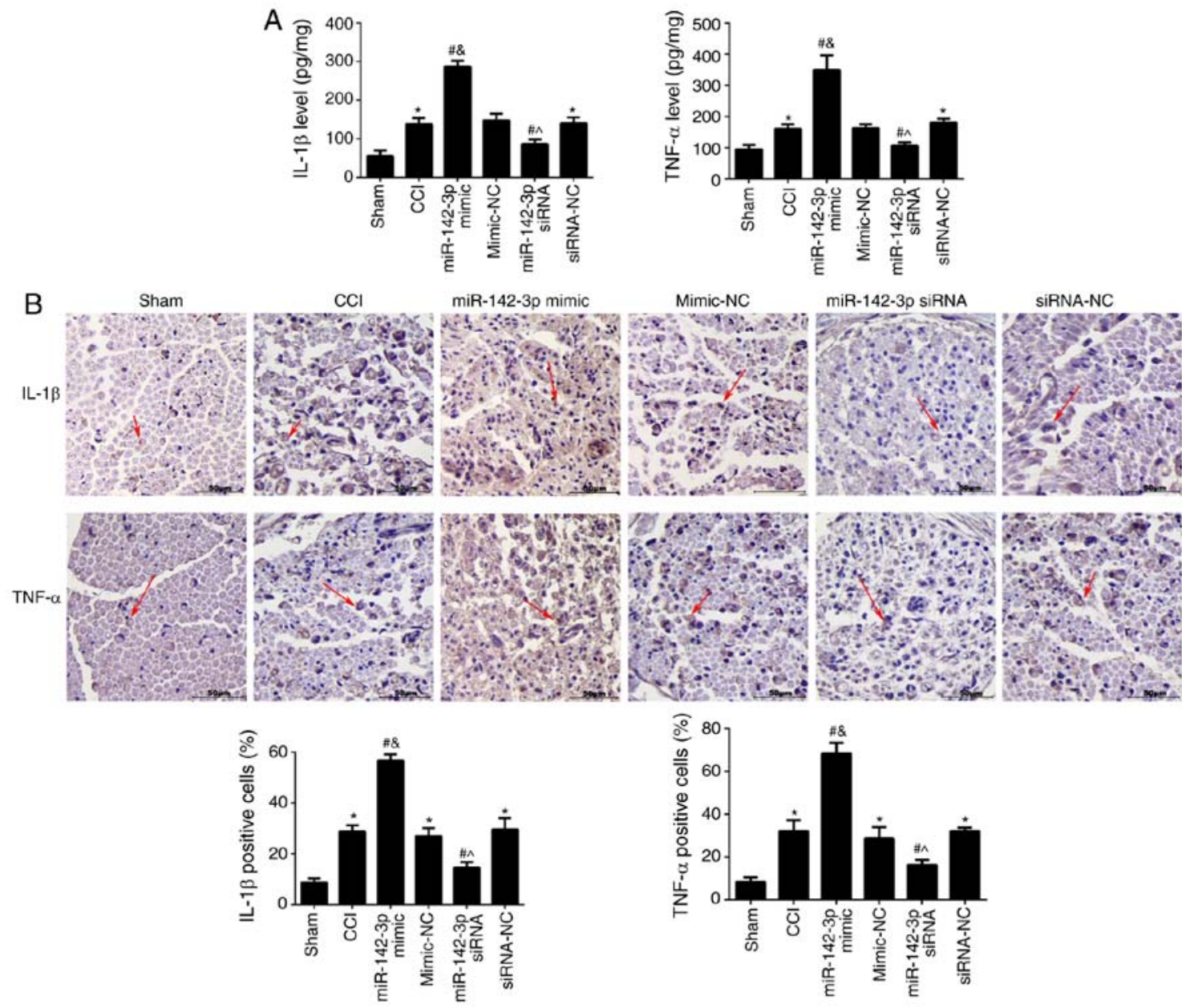

Figure 3. Effects of miR-142-3p on inflammatory cytokines following sciatic nerve injury. (A) The levels of IL-1 $\beta$ and TNF- $\alpha$ were measured by ELISA. (B) The expression of IL- $1 \beta$ (red arrows) and TNF- $\alpha$ (red arrows) was analysed by immunohistochemistry. Scale bar, $50 \mu \mathrm{m} .{ }^{*} \mathrm{P}<0.05$ vs. sham group. ${ }^{*} \mathrm{P}<0.05$ vs. CCI group. ${ }^{\circledR} \mathrm{P}<0.05$ vs. mimic-NC group. ${ }^{\wedge} \mathrm{P}<0.05$ vs. siRNA-NC group. Each experiment was repeated five times. CCI, chronic compression injury; $\mathrm{NC}$, negative control; siRNA, small interfering RNA; miR, microRNA.

IL-1 $\beta$ and TNF- $\alpha$ in the sciatic nerve was analysed using ELISA (Fig. 3A) and immunohistochemistry (Fig. 3B). The results showed that the contents of IL- $1 \beta$ and TNF- $\alpha$ in the CCI group were significantly higher compared with the sham group $(\mathrm{P}<0.05)$. Compared with the CCI group, miR-142-3p overexpression significantly increased the levels of IL-1 $\beta$ and TNF- $\mathrm{a}(\mathrm{P}<0.05)$, while miR-142-3p-silencing showed opposite effects $(\mathrm{P}<0.05)$. These results revealed that miR-142-3p-overexpression increased the secretion of inflammatory cytokines in rats with sciatic nerve injury.

Expression of AC9 in sciatic pain and the AMPK pathway. RT-qPCR was used to analyse AC9 expression in the L4-6 sciatic nerve (Fig. 4A). Following the injection of pcDNA-AC9, AC9 expression was significantly increased $(\mathrm{P}<0.05)$, whereas injection of AC9 siRNA significantly decreased AC9 expression $(\mathrm{P}<0.05)$ compared with the CCI groups. The cAMP content was significantly decreased in the AC9 siRNA group, but significantly increased in the pcDNA-AC9 group compared with the CCI group ( $\mathrm{P}<0.05$; Fig. 4B).

The results from PWMT and PWTL analysis (Fig. 4C) indicated that AC9 overexpression effectively alleviated sciatic pain following surgery, while AC9 silencing obviously exacerbated sciatic pain following surgery compared with the
CCI group $(\mathrm{P}<0.05)$. These results suggested that AC9 downregulation leads to neuropathic pain in CCI rats.

The levels of $p$-AMPK/AMPK, $p-N F-\kappa B / N F-\kappa B$ and $\mathrm{p}-\mathrm{I} \kappa \mathrm{Ba} / \mathrm{I} \kappa \mathrm{Ba}$ in the sciatic nerve were detected using western blotting (Fig. 4D). Following pcDNA-AC9 transfection, compared with the CCI group, the phosphorylation levels of AMPK and IкBa were significantly increased $(\mathrm{P}<0.05)$, while the phosphorylation levels of NF- $\mathrm{BB}$ was further decreased $(\mathrm{P}<0.05)$. However, transfection of AC9 siRNA significantly decreased the phosphorylation levels of AMPK and IкBa, and increased those of NF- $\mathrm{kB}$, as compared with the $\mathrm{CCI}$ group $(\mathrm{P}<0.05)$.

Effects of AC9 on inflammatory cytokine content following sciatic nerve injury. The levels of IL- $1 \beta$ and TNF- $\alpha$ were measured using ELISA (Fig. 5A) and immunohistochemistry (Fig. 5B) to analyse the effects of AC9 on IL- $1 \beta$ and TNF- $\alpha$ in the sciatic nerve of each group. AC9 overexpression significantly reduced the expression of IL- $1 \beta$ and TNF-a $(\mathrm{P}<0.05)$, while AC9 silencing significantly increased the contents of IL-1 $\beta$ and TNF-a compared with the CCI group $(\mathrm{P}<0.05)$. These results revealed that $\mathrm{AC} 9$ overexpression suppressed the secretion of inflammatory cytokines in rats with sciatic nerve injury. 
A

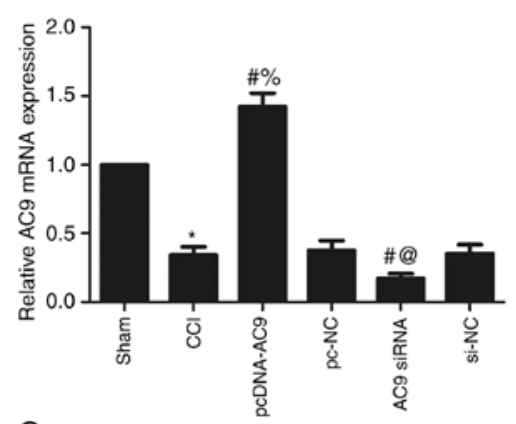

C

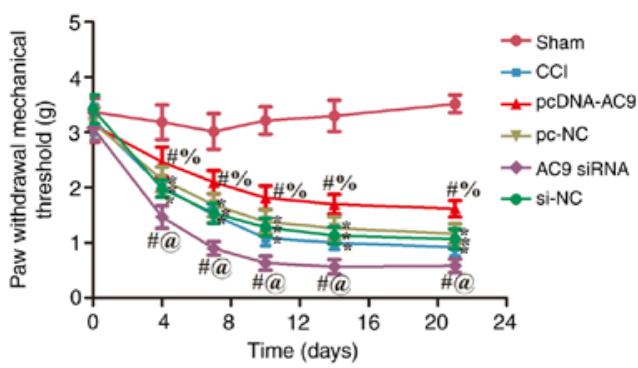

B

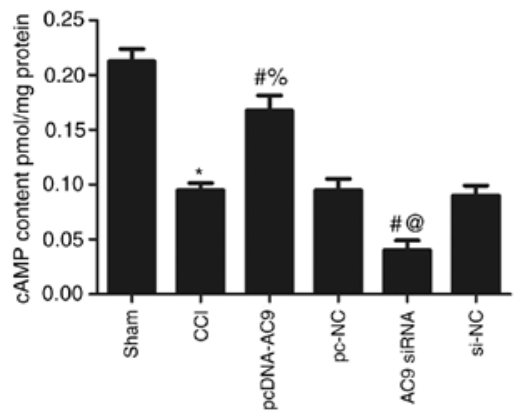

D
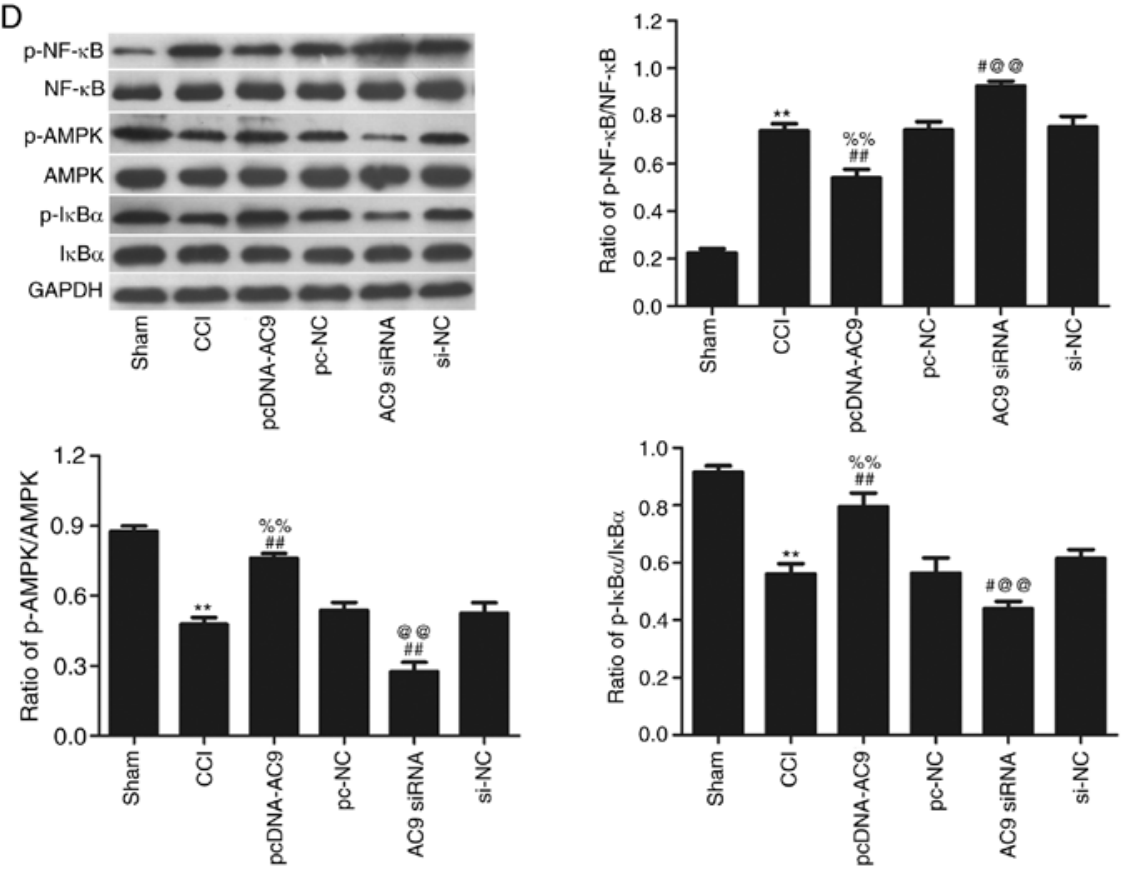

Figure 4. Effects of AC9 on sciatic pain and the AMPK pathway. (A) The expression of miR-142-3p was analysed by reverse transcription-quantitative PCR. (B) cAMP levels were analysed by ELISA. (C) The changes in paw withdrawal mechanical threshold and paw withdrawal thermal latency were measured in

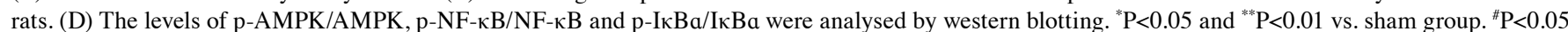
and ${ }^{\# \#} \mathrm{P}<0.01$ vs. CCI group. ${ }^{\%} \mathrm{P}<0.05$ and ${ }^{\% \%} \mathrm{P}<0.01$ vs. mimic-NC group. ${ }^{\circledR} \mathrm{P}<0.05$ and ${ }^{\circledR}{ }^{\circledR} \mathrm{P}<0.01$ vs. siRNA-NC group. Each experiment was repeated five times. AMPK, AMP-activated protein kinase; NC, negative control; siRNA, small interfering RNA; CCI, chronic compression injury; pc-NC, pcDNA-AC9 control vector; AC9, adenylate cyclase 9.

AC9 is the target gene of $m i R-142-3 p$. AC9 was predicted as a candidate target gene of miR-142-3p using TargetScan (http://www.targetscan.org; Fig. 6A). Next, the present study confirmed whether miR-142-3p is directly binds to the 3'-UTR of AC9. The AC9 3'-UTR containing wild-type or mutated miR-142-3p target site in a firefly luciferase reporter vector was cloned to obtain the reporter plasmids wt-AC9 or mut-AC9, respectively. When miR-142-3p mimic was co-transfected with wt-AC9, the luciferase activity was significantly attenuated, whereas a reversal in luciferase expression was observed when miR-142-3p mimic was co-transfected with mut-AC9 (Fig. 6B).
The transfection efficiency of miR-142-3p in each group was analysed by RT-qPCR (Fig. 6C). miR-142-3p expression was significantly increased in the miR-142-3p mimic group and significantly decreased in the miR-142-3p siRNA group when compared with their respective $\mathrm{NC}$ groups $(\mathrm{P}<0.05)$. RT-qPCR and western blotting (Fig. 6D) results showed that miR-142-3p-overexpression significantly reduced AC9 expression, and miR-142-3p silencing significantly increased AC9 expression $(\mathrm{P}<0.05)$. These data provided evidence that miR-142-3p could inhibit AC9 expression by directly binding to the AC9 3'-UTR. 


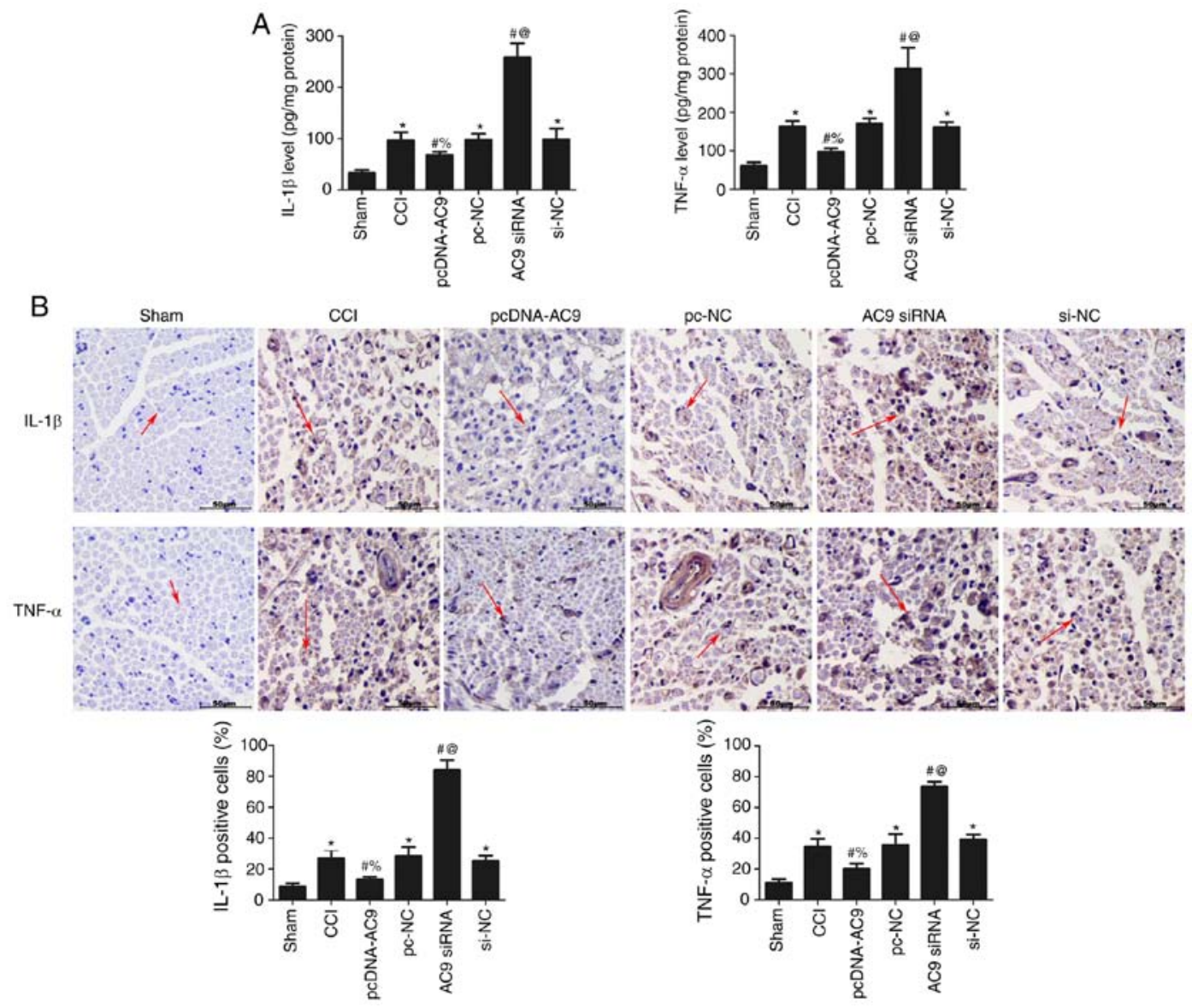

Figure 5. Effects of AC9 on inflammatory cytokines following sciatic nerve injury. (A) The levels of IL-1 $\beta$ and TNF- $\alpha$ were measured by ELISA. (B) The expression of IL-1 $\beta$ (red arrows) and TNF- $\alpha$ (red arrows) were analysed by immunohistochemistry. Scale bar, $50 \mu \mathrm{m}$. ${ }^{*} \mathrm{P}<0.05$ vs. sham group. ${ }^{~} \mathrm{P}<0.05$ vs. CCI group. ${ }^{\%} \mathrm{P}<0.05$ vs. mimic-NC group. ${ }^{\circledR} \mathrm{P}<0.05$ vs. siRNA-NC group. Each experiment was repeated five times. CCI, chronic compression injury; siRNA, small interfering RNA; NC, negative control; pc-NC, pcDNA-AC9 control vector; AC9, adenylate cyclase 9.

\section{A}

AC9 3'UTR-WT: 5'-...UUCUUUUCUUAAAAAACACUACC...-3' miR-142-3p: 3'-...AGGUAUUUCAUCCUUUGUGAUGU...-5 AC9 3'UTR-WT: $\quad$ '-...UUCUUUUCUUAAAAAUGUGAUGC..-3'

$$
\text { B }
$$

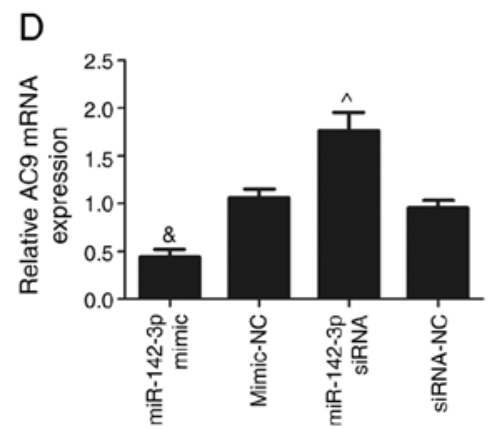

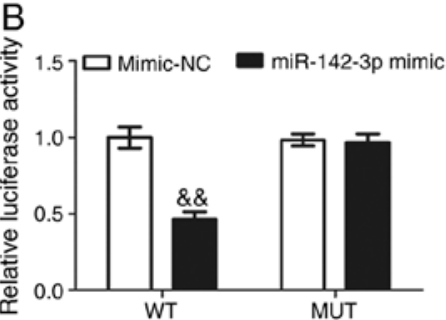
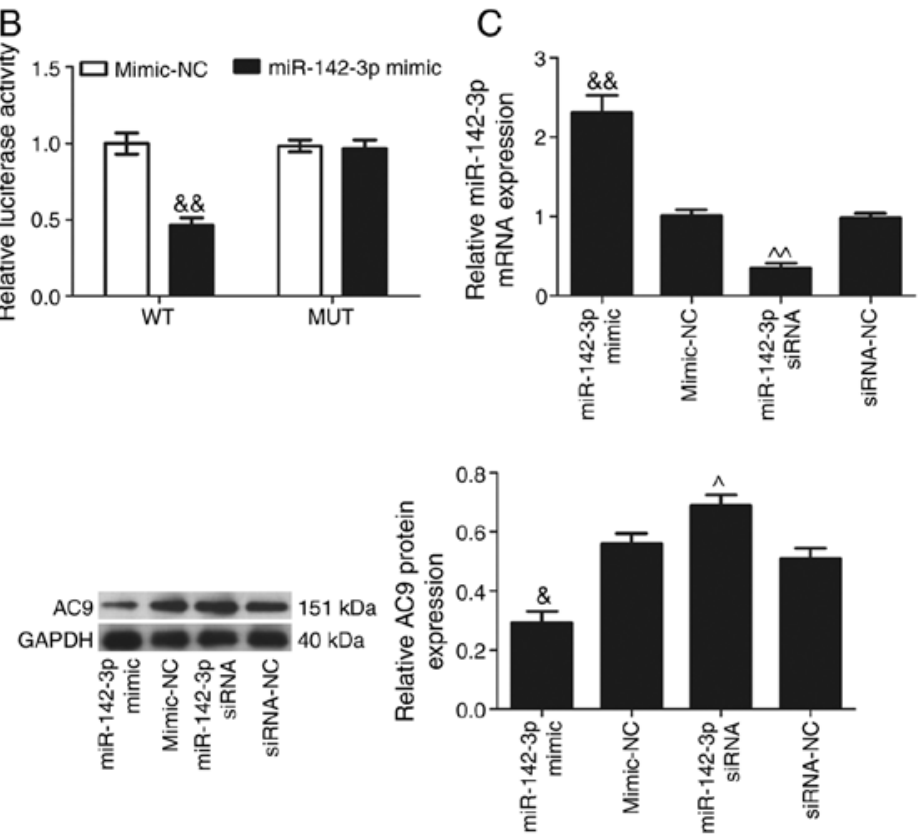

Figure 6. AC9 is the target gene of miR-142-3p. (A) AC9 was predicted as a candidate target gene of miR-142-3p using TargetScan (http://www.targetscan. org). (B) Luciferase reporter assay analysis of miR-142-3p overexpression on the activity of WT and MUTAC9 at the 3'-UTR. \&\&P<0.05 vs. mimic-NC group. (C) RT-qPCR showing the transfection efficiency of miR-142-3p in each group. (D) RT-qPCR and western blotting showing the effects of miR-142-3p on AC9 expression. ${ }^{\&} \mathrm{P}<0.05$ and ${ }^{\&}{ }^{\&} \mathrm{P}<0.01$ vs. mimic-NC group. ${ }^{\wedge} \mathrm{P}<0.05$ and ${ }^{\wedge} \mathrm{P}<0.01$ vs. siRNA-NC group. Each experiment was repeated three times. miR, microRNA; RT-qPCR, reverse transcription-quantitative PCR; siRNA, small interfering RNA; NC, negative control; AC9, adenylate cyclase 9; WT, wild-type; MUT, mutant; 3'-UTR, 3'-untranslated region. 

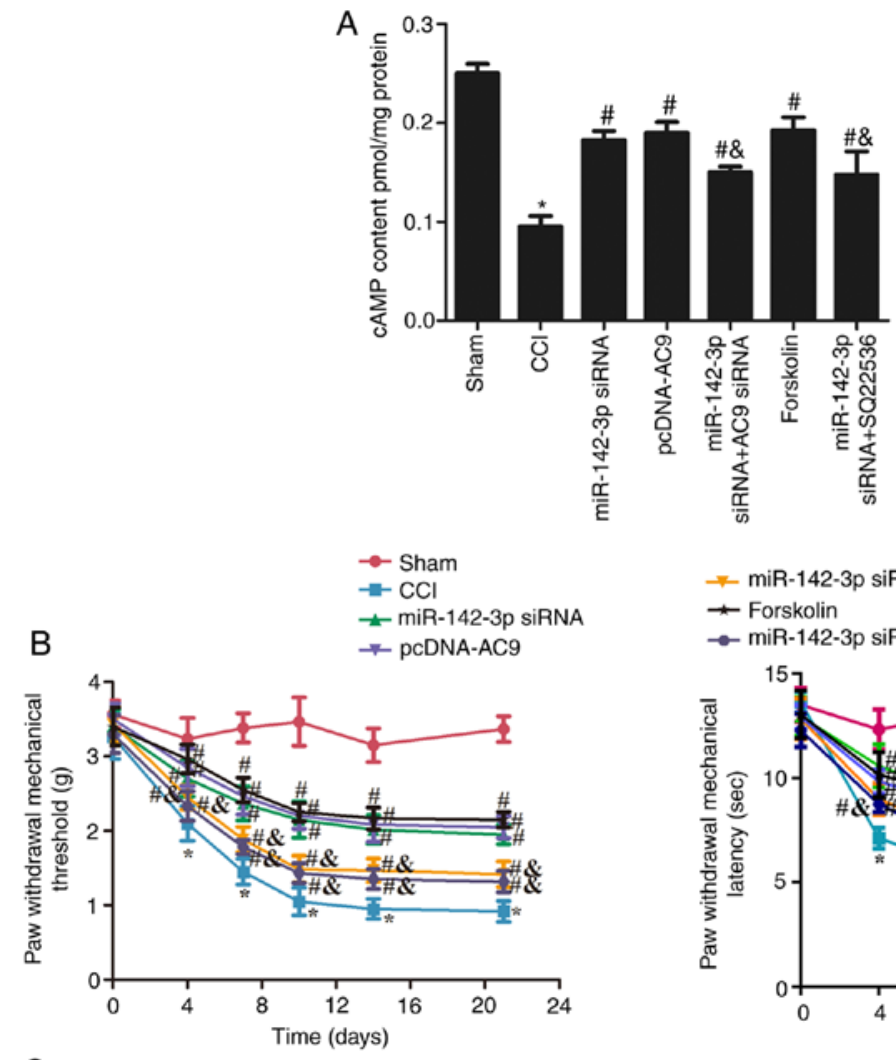

$$
\begin{aligned}
& \text { — miR-142-3p siRNA+AC9 siRNA } \\
& \text { - Forskolin } \\
& \rightarrow \text { miR-142-3p siRNA+SQ22536 }
\end{aligned}
$$

C
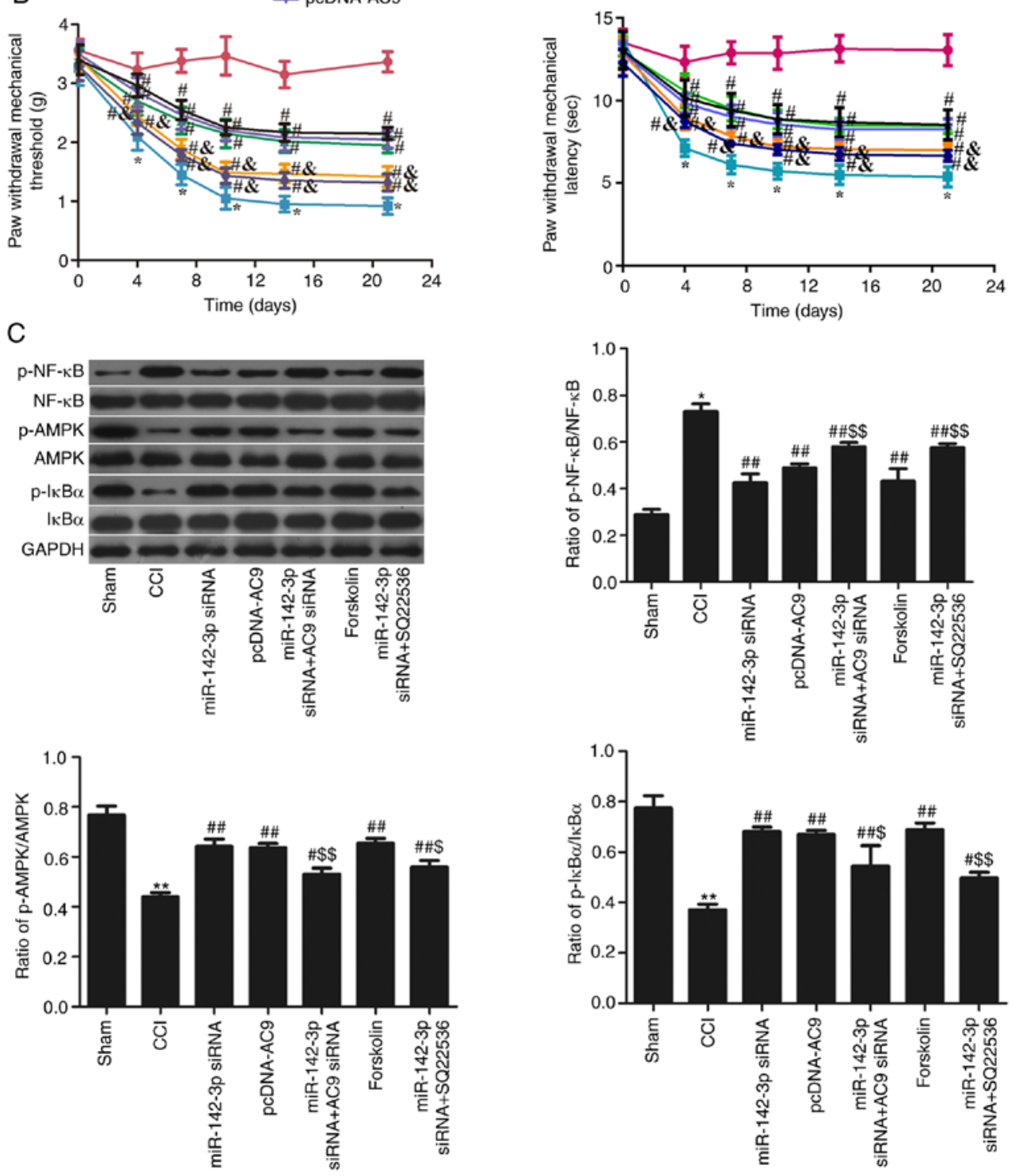

Figure 7. miR-142-3p regulates sciatic pain via the AC9/cAMP/AMPK axis in rats with sciatic nerve injury. (A) cAMP levels were analysed by ELISA. (B) Changes in paw withdrawal mechanical threshold and paw withdrawal thermal latency were measured in rats. (C) The levels of p-AMPK/AMPK,

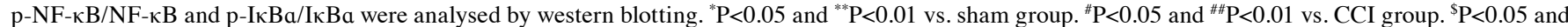
${ }^{\$ \$} \mathrm{P}<0.05$ vs. miR-142-3p siRNA group. ${ }^{\circledR} \mathrm{P}<0.05$ vs. pcDNA-AC9 group. Each experiment was repeated five times. miR, microRNA; CCI, chronic compression injury; p, phosphorylated; AC9, adenylate cyclase 9; AMPK, AMP-activated protein kinase; siRNA, small interfering RNA.

miR-142-3p regulates sciatic pain via the AC9/cAMP/AMPKA axis in rats with sciatic nerve injury. Following simultaneous intrathecal injection of miR-142-3p siRNA and AC9 siRNA, cAMP levels were significantly decreased compared 

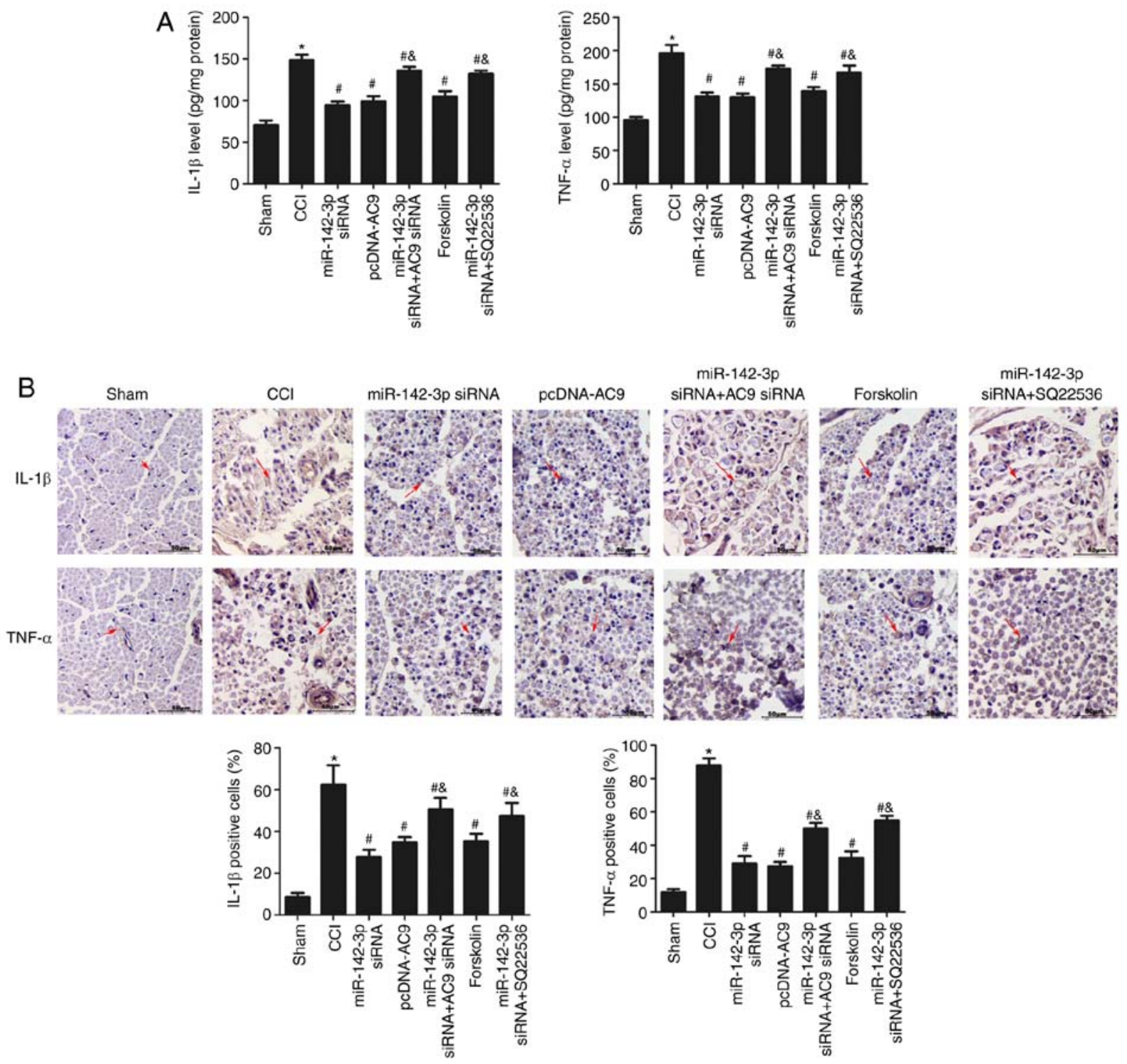

Figure 8. miR-142-3p regulates inflammatory cytokines via the AC9/cAMP/AMPK axis in rats with sciatic nerve injury. (A) The levels of IL-1 $\beta$ and TNF- $\alpha$ were measured by ELISA. (B) The expression of IL-1 $\beta$ (red arrows) and TNF- $\alpha$ (red arrows) was analysed by immunohistochemistry. Scale bar, $50 \mu \mathrm{m}$. ${ }^{*} \mathrm{P}<0.05$ vs. sham group. ${ }^{*} \mathrm{P}<0.05$ vs. CCI group. ${ }^{\circledR} \mathrm{P}<0.05$ vs. pcDNA-AC9 group. Each experiment was repeated five times. miR, microRNA; CCI, chronic compression injury; AMPK, AMP-activated protein kinase; siRNA, small interfering RNA.

with intrathecal injection of miR-142-3p siRNA or AC9 pcDNA-AC9 alone ( $\mathrm{P}<0.05$; Fig. 7A). In addition, the administration of cAMP activator forskolin significantly increased cAMP levels compared with the sham group $(\mathrm{P}<0.05)$. Following simultaneous intrathecal injection of miR-142-3p siRNA and cAMP inhibitor (SQ22536), cAMP levels significantly decreased compared with the miR-142-3p siRNA group $(\mathrm{P}<0.05)$. Fig. 7B showed that the simultaneous intrathecal injection of miR-142-3p siRNA and AC9 siRNA significantly decreased the PWMT and PWTL values $(\mathrm{P}<0.05)$ compared with the miR-142-3p siRNA group $(\mathrm{P}<0.05)$. Following forskolin administration, the PWMT and PWTL values were increased compared with the CCI group $(\mathrm{P}<0.05)$. Following the simultaneous intrathecal injection of miR-142-3p siRNA and SQ22536, the PWMT and PWTL values were decreased compared with the miR-142-3p siRNA group $(\mathrm{P}<0.05)$. As shown in Fig. 7C, the levels of AMPK pathway-related proteins were measured. Compared with the miR-142-3p siRNA group, the levels of p-AMPK and p-IкBa decreased, and p-NF-кB p65 levels increased in the miR-142-3p siRNA + AC9 siRNA group $(\mathrm{P}<0.05)$. Compared with the CCI group, forskolin significantly increased the levels of p-AMPK and p-IкBa and reduced that of $\mathrm{p}-\mathrm{NF}-\mathrm{kB}$ p65 $(\mathrm{P}<0.05)$. The levels of p-AMPK and $\mathrm{p}-\mathrm{I} \kappa \mathrm{Ba}$ were significantly reduced, and that of $\mathrm{p}-\mathrm{NF}-\mathrm{\kappa B}$ p65 was increased in the miR-142-3p siRNA and SQ22536 groups compared with the miR-142-3p siRNA group $(\mathrm{P}<0.05)$. These data indicated that miR-142-3p silencing reduced neuropathic pain in CCI rats by upregulating AC9/cAMP/AMPK.

miR-142-3p regulates inflammatory cytokines via the AC9/cAMP/AMPKA axis in rats with sciatic nerve injury. In addition, inflammatory factors were analysed in each group using ELISA (Fig. 8A) and immunohistochemistry (Fig. 8B). As shown in Fig. 8A, compared with the miR-142-3p siRNA group, the expression of IL- $1 \beta$ and TNF-a significantly decreased in the miR-142-3p siRNA + AC9 siRNA group 
$(\mathrm{P}<0.05)$. Compared with the CCI group, forskolin administration reduced the expression of these inflammatory cytokines in the sciatic nerve of CCI rats $(\mathrm{P}<0.05)$, and no differences were observed among the miR-142-3p siRNA, pcDNA-AC9 and forskolin groups $(\mathrm{P}>0.05)$, while the levels of IL-1 $\beta$ and TNF-a were significantly higher in the miR-142-3p siRNA + SQ22536 group compared with the miR-142-3p siRNA group. As shown in Fig. 8B, the immunohistochemical results were consistent with the ELISA results, indicating that miR-142-3p silencing restrained inflammatory cytokine expression via the AC9/cAMP/AMPKA axis in rats with sciatic nerve injury.

\section{Discussion}

In the present study, it was found that miR-142-3p was highly expressed, but AC9 was lowly expressed in the sciatic nerve of CCI rats. miR-142-3p inhibition increased the expression of AC9 and cAMP in the sciatic nerve of CCI rats. Furthermore, the inhibition of miR-142-3p activated AMPK activity and inhibited the release of IL-1 $\beta$ and TNF- $a$ in the sciatic nerve of CCI rats. AC9 exerted the opposite effects in rats with sciatic nerve injury compared to miR-142-3p. miR-142-3p could inhibit AC9 expression by directly binding to the AC9 3'-UTR. Consequently, these results suggested that miR142-3p targets AC9 to reduce sciatic nerve injury-induced neuropathic pain by regulating the cAMP/AMPK signalling pathway.

Neuropathic pain often results from disease or injury which influences the steady state of the nervous or central nervous system (21). For the past few years, miRNAs have gradually emerged as the functional regulators of consistent neuropathic pain conception and development (22). Increasing evidence has shown that miRNA-based medical treatment may be an effective means of blocking neuropathic pain $(4,23)$. A study revealed that miR-195 induced by spinal nerve ligation-aggravated neuropathic pain by inhibiting Atg14 (24). At the same time, miR-221, miR-19a and miR-155 alleviated neuropathic pain by targeting the suppressor of cytokine signalling (25-27). Studies have demonstrated that miR-146a-5p overexpression lessened neuropathic pain by suppressing TNF receptor-associated factor-6-mediated neuro-inflammation (28). miR-144, miR-132-3p, miR-124a and miR-15b have been shown to be involved in the process of neuropathic pain $(25,28,29)$. Evidence has suggested that miRNAs are emerging as novel and promising factors for neuropathic treatment. A previous study demonstrated that miR-142-3p served as a key miRNA for relieving neuropathic pain (5). Another study also revealed that miR-142-3p targets high mobility group box 1 to positively regulate neuropathic pain (30). However, Wang et al (6) found that $\mathrm{miR}-142-3 \mathrm{p}$ played a negative role in sciatic nerve conditioning injury by regulating the AC9/cAMP axis. The present study also demonstrated that miR-142-3p expression was higher in rats with sciatic nerve injury.

Previous studies have indicated that the AMPK-related signalling pathway plays an essential role in the biology of neuropathic pain $(13,14)$. The underlying mechanism focuses on AMPK-driven protein synthesis. The present study also demonstrated that AMPK activity is activated by the increased miR-142-3p expression. Other studies have revealed that AMPK activation reduced mechanical hypersensitivity by restraining the expression of inflammatory cytokines in a rat model of neuropathic pain, which is linked to increased IL-1 $\beta$, IL-6 and IL-8 expression $(31,32)$. The present study demonstrated that miR-142-3p overexpression increased the production of TNF- $\alpha$ and IL-1 $\beta$ in sciatic nerve injury rats, supporting a pro-inflammatory role of miR-142-3p.

However, the present study had several limitations. For example, the cellular co-expression of miR-142-3p and AC9 in the sciatic nerve has not been confirmed, and only the NF- $\kappa \mathrm{B}$ p65 subunit was analyzed; other subunits need to be analyzed as well, such as protein kinase A and mitogen-activated protein kinase. In addition, whether there is any difference between the ipsilateral and contralateral sciatic nerve also remains to be studied. Neuropathic pain may be associated with multiple chronic diseases, which exist in the balance of homeostasis.

In conclusion, miR-142-3p is highly expressed while AC9 is lowly expressed in rats with sciatic nerve injury. miR-142-3p inhibition could increase the expression of AC9 and $\mathrm{cAMP}$, and further activate the activity of AMPK. The miR-142-3p/AC9/cAMP/AMPK axis can be used as an important signaling pathway for sensory function recovery following sciatic nerve injury.

\section{Acknowledgements}

Not applicable.

\section{Funding}

No funding was received.

\section{Availability of data and materials}

All data generated or analyzed during this study are included in this published article or are available from the corresponding author on reasonable request.

\section{Authors' contributions}

XL and SW performed experimental work and the data collection and interpretation. XL and XY participated in the design and coordination of the experimental work and acquisition of the data. SW and XY participated in the study design, data collection and data analysis. XL and HC designed the study, analysed the data and drafted the manuscript. All authors read and approved the final manuscript.

\section{Ethics approval and consent to participate}

This study was approved by the Institutional Animal Care and Use Committee of Yantaishan Hospital (approval no. 20180705-0012).

\section{Patient consent for publication}

Not applicable.

\section{Competing interests}

The authors declare that they have no competing interests. 


\section{References}

1. Baron R: Peripheral neuropathic pain: From mechanisms to symptoms. Clin J Pain 16: (Suppl 2): S12-S20, 2000.

2. Baron R: Mechanisms of disease: Neuropathic pain-a clinical perspective. Nat Clin Pract Neurol 2: 95-106, 2006.

3. O'Connor AB and Dworkin RH: Treatment of neuropathic pain: An overview of recent guidelines. Am J Med 122 (Suppl 10) S22-S32, 2009.

4. Jiangpan P, Qingsheng M, Zhiwen Y and Tao Z: Emerging role of microRNA in neuropathic pain. Curr Drug Metab 17: 336-344, 2016.

5. Lu X, Li X, He Q, Gao J, Gao Y, Liu B and Liu F: miR-142-3p regulates the formation and differentiation of hematopoietic stem cells in vertebrates. Cell Res 23: 1356-1368, 2013.

6. Wang T, Li B, Wang Z, Wang X, Xia Z, Ning G, Wang X, Zhang Y, Cui L, Yu M, et al: Sorafenib promotes sensory conduction function recovery via miR-142-3p/AC9/cAMP axis post dorsal column injury. Neuropharmacology 148: 347-357, 2019.

7. Ouyang B, Tang Z, Hou X, Chen D, Guo Q and Weng Y: Trichostatin A suppresses up-regulation of histone deacetylase 4 and reverses differential expressions of miRNAs in the spinal cord of rats with chronic constrictive injury. Nan Fang Yi Ke Da Xue Xue Bao 39: 1421-1426, 2019 (In Chinese).

8. Tahamtan A, Teymoori-Rad M, Nakstad B and Salimi V: Anti-inflammatory MicroRNAs and their potential for inflammatory diseases treatment. Front Immunol 9: 1377, 2018.

9. Ji RR, Nackley A, Huh Y, Terrando $\mathrm{N}$ and Maixner W: Neuroinflammation and central sensitization in chronic and widespread pain. Anesthesiology 129: 343-366, 2018.

10. Sakaue G, Shimaoka M, Fukuoka T, Hiroi T, Inoue T, Hashimoto N, Sakaguchi T, Sawa Y, Morishita R, Kiyono H, et al: NF-kappa B decoy suppresses cytokine expression and thermal hyperalgesia in a rat neuropathic pain model. Neuroreport 12 : 2079-2084, 2001.

11. Scholz J and Woolf CJ: The neuropathic pain triad: Neurons, immune cells and glia. Nat Neurosci 10: 1361-1368, 2007.

12. Price TJ and Dussor G: AMPK: An emerging target for modification of injury-induced pain plasticity. Neurosci Lett 557: 9-18, 2013.

13. Lu L, Pan C, Chen L, Hu L, Wang C, Han Y, Yang Y, Cheng Z and Liu WT: AMPK activation by peri-sciatic nerve administration of ozone attenuates CCI-induced neuropathic pain in rats. J Mol Cell Biol 9: 132-143, 2017.

14. Huang ZJ, Li HC, Cowan AA, Liu S, Zhang YK and Song XJ: Chronic compression or acute dissociation of dorsal root ganglion induces cAMP-dependent neuronal hyperexcitability through activation of PAR2. Pain 153: 1426-1437, 2012.

15. Bennett GJ and Xie YK: A peripheral mononeuropathy in rat that produces disorders of pain sensation like those seen in man. Pain 33: 87-107, 1988.

16. Pan Z, Shan Q, Gu P, Wang XM, Tai LW, Sun M, Luo X, Sun L and Cheung CW: miRNA-23a/CXCR4 regulates neuropathic pain via directly targeting TXNIP/NLRP3 inflammasome axis. J Neuroinflammation 15: 29, 2018.

17. Aglah C, Gordon T and Posse de Chaves EI: cAMP promotes neurite outgrowth and extension through protein kinase A but independently of Erk activation in cultured rat motoneurons. Neuropharmacology 55: 8-17, 2008.
18. Hargreaves K, Dubner R, Brown F, Flores C and Joris J: A new and sensitive method for measuring thermal nociception in cutaneous hyperalgesia. Pain 32: 77-88, 1988.

19. Shao H, Xue Q, Zhang F, Luo Y, Zhu H, Zhang X, Zhang H, Ding W and Yu B: Spinal SIRT1 activation attenuates neuropathic pain in mice. PLoS One 9: e100938, 2014.

20. Livak KJ and Schmittgen TD: Analysis of relative gene expression data using real-time quantitative PCR and the 2(-Delta Delta C(T)) method. Methods 25: 402-408, 2001.

21. Tsuda M: Microglia in the spinal cord and neuropathic pain. J Diabetes Investig 7: 17-26, 2016.

22. Andersen HH, Duroux M and Gazerani P: MicroRNAs as modulators and biomarkers of inflammatory and neuropathic pain conditions. Neurobiol Dis 71: 159-168, 2014.

23. Dai Z, Chu H, Ma J, Yan Y, Zhang X and Liang Y: The regulatory mechanisms and therapeutic potential of MicroRNAs: From chronic pain to morphine tolerance. Front Mol Neurosci 11: 80, 2018.

24. Shi G, Shi J, Liu K, Liu N, Wang Y, Fu Z, Ding J, Jia L and Yuan W: Increased miR-195 aggravates neuropathic pain by inhibiting autophagy following peripheral nerve injury. Glia 61: 504-512, 2013.

25. Heyn J, Luchting B, Hinske LC, Hübner M, Azad SC and Kreth S: miR-124a and miR-155 enhance differentiation of regulatory $\mathrm{T}$ cells in patients with neuropathic pain. J Neuroinflammation 13: $248,2016$.

26. Xia L, Zhang Y and Dong T: Inhibition of MicroRNA-221 alleviates neuropathic pain through targeting suppressor of cytokine signaling 1. J Mol Neurosci 59: 411-420, 2016.

27. Wang C, Jiang Q, Wang M and Li D: miR-19a targets suppressor of cytokine signaling 1 to modulate the progression of neuropathic pain. Int J Clin Exp Pathol 8: 10901-10907, 2015.

28. Lu Y, Cao DL, Jiang BC, Yang T and Gao YJ: MicroRNA-146a-5p attenuates neuropathic pain via suppressing TRAF6 signaling in the spinal cord. Brain Behav Immun 49: 119-129, 2015.

29. Leinders M, Üceyler N, Pritchard RA, Sommer C and Sorkin LS: Increased miR-132-3p expression is associated with chronic neuropathic pain. Exp Neurol 283: 276-286, 2016.

30. Zhang Y, Mou J, Cao L, Zhen S, Huang H and Bao H: MicroRNA-142-3p relieves neuropathic pain by targeting high mobility group box 1. Int J Mol Med 41: 501-510, 2018.

31. Xiang HC, Lin LX, Hu XF, Zhu H, Li HP, Zhang RY, Hu L, Liu WT, Zhao YL, Shu Y, et al: AMPK activation attenuates inflammatory pain through inhibiting NF-kappaB activation and IL-1beta expression. J Neuroinflammation 16: 34, 2019.

32. Hasanvand A, Amini-Khoei H, Hadian MR, Abdollahi A, Tavangar SM, Dehpour AR, Semiei E and Mehr SE: Anti-inflammatory effect of AMPK signaling pathway in rat model of diabetic neuropathy. Inflammopharmacology 24: 207-219, 2016.

This work is licensed under a Creative Commons Attribution-NonCommercial-NoDerivatives 4.0 International (CC BY-NC-ND 4.0) License. 